\title{
Abstracts
}

\section{Transition/Adult Care Session 1}

\author{
Evaluation of Spina Bifida Transitional Care \\ Practices in the US \\ Kelly, Maryellen S. ${ }^{1}$, Thibadeau, Judy ${ }^{2}$, Struwe, Sara ${ }^{3}$, \\ Ramen, Lisa ${ }^{3}$, Ouyang, Lijing ${ }^{2}$, Routh, Jonathan ${ }^{1}$ \\ ${ }^{1}$ Duke University \\ ${ }^{2}$ Centers for Disease Control and Prevention \\ ${ }^{3}$ Spina Bifida Association
}

Background Recent studies have revealed that the lack of continuity and preparedness of adult-centered care for those with Spina Bifida may have detrimental health consequences. At current state, little is known regarding the state of transitional care management in the US. We sought to describe current practices of transitional care services offered at Spina Bifida clinics in the US.

Methods Survey design followed the validated transitional care survey by the National Cystic Fibrosis center. Survey was amended for Spina Bifida. Face validity was completed. Survey was distributed to registered clinics via the Spina Bifida Association. Results were analyzed via descriptive means.

Results Total of 27 clinics responded. Over 90 characteristics were analyzed per clinic. $68 \%$ of clinics do not have a written protocol for transition. Over $50 \%$ do not routinely evaluate their process. Less than $50 \%$ discuss insurance coverage changes with patients. Only $30 \%$ communicate with the adult providers. Sexuality, pregnancy and reproductive issues are not readily discussed in most clinics. The concept of transition is discussed with patients over $75 \%$ of the time and mobility, bowel and bladder management, weight, alcohol and drug use, are discussed in nearly all clinics.

$40 \%$ of clinics have a list of desired skills/knowledge for a patient to obtain. Overall clinics self-rate themselves as a 5/10 in their ability to provide services for their patients during transition.

Conclusions Characteristics of current transitional care services and formal transitional care programs at
US clinics show wide variances in what is offered to patients and families.

\author{
A Transition Protocol at Children's Hospital of \\ Pittsburgh of UPMC \\ Akamagwuna, Unoma O., Young, Rachel, Houtrow, \\ Amy, Dicianno, Brad E. \\ University of Pittsburgh
}

Background Transitioning from pediatric to adult health care is challenging, especially for those with disabilities such as Spina Bifida. To assist with the preparation to transition the Children's Hospital of Pittsburgh's Spina Bifida Program developed an ongoing quality improvement initiative.

Methods The Program serves individuals with spinal disorders from birth to age 22 when they are transitioned to the adult Spina Bifida Program. The quality improvement initiative uses the guidelines by GotTransition.org to institute a transition and education program. At each visit core topics are addressed including diet, exercise, self-management, community engagement, puberty, sex education and vocation discussed at an appropriate developmental level. Upon graduation patients receive an education letter for health providers, a Personal Health Record, and their first appointment in the Adult Spina Bifida Clinic. Transition readiness of patients and families is tracked via a transition readiness tool available on GotTransition.org.

Results In the past 5 years, 43 patients have have graduated, $67 \%$ were seen in the adult clinic and $33 \%$ were lost to follow up. Over $50 \%$ knew the basics about their medical condition and were able to identify where they go to get care. $60 \%$ indicated a lack of knowledge of health privacy laws and how to navigate the healthcare system. $75 \%$ identified they need to learn how their health privacy changed at 18 . Post transition, only $37 \%$ of young adult's surveyed reported that they had ever spent time alone with their health providers. 
Conclusions The initial results show that young adults have confidence about their medical history. However the majority identify a lack of knowledge of the healthcare system and how their autonomy changes as an adult. This highlights that more needs to be done in these areas as part of our transition process. The data also demonstrated that young adult health privacy was not prioritized.

2016 NEO SBA Adult Ed Day: Building a transition collaborative for SB Y and YA to create seamless access to adult based services

Fortuna, Suzanne M. ${ }^{1,2}$, Zeni, Mary Beth ${ }^{2}$

${ }^{1}$ UHCMC/RBC Hospital

${ }^{2}$ Ursuline College

Background Many Pediatric Myelo teams exist within the 8 Ohio children's hospitals. Yet less than 10 adult programs exist across the US. The Spina Bifida population is increasingly living into adulthood. Lack of coordinated adult transition teams and clinics necessitates education to foster collaboration with community and subspecialty providers. A one-day Summit was planned, implemented, and evaluated with the aim to provide seamless transition from pediatric to adult services for youth and young adults with Spina Bifida. Methods Conference attendees were requested to complete a pre and post-conference survey about youth/ young adult transition practices and beliefs. Attendees also completed a program evaluation. Study protocol was approved by the college Institutional Review Board.

Results Twenty-nine participants attended the Educational Summit. Fourteen speakers provided content about transition from pediatric to adult programs, transition obstacles and challenges, mental health assessments, and bowel program innovations. Breakout sessions for sexuality were also provided. Sponsorship was provided by 4 vendors. A majority of attendees (21 of 29 , or $72 \%$ ) were registered nurses or advanced practice registered nurses who work with youth and families living with Spina Bifida. Participants reported increased awareness and commitment to transition practice. Wilcoxon signed rank test was used to test significance (alpha $=0.05$ ) between pre and post conference surveys; significance was found for all 13 questions addressing transition practices and beliefs. Conclusions The SBA Educational Summit on transition for youth and young adults living with Spina Bifida was successful and many requested another op- portunity to attend future transition events. The Educational Summit met the aim to educate participants to commit and contribute to active transition planning and assistance in their practices.

\section{Descriptive Analysis and Profile of Health Care Transition (HCT) Services Provided to Adolescents and Emerging Adults (AEA) in the Movin' On Up HCT Program \\ Betz, Cecily L. ${ }^{1,2}$, Smith, Kathryn ${ }^{1,2}$, \\ Van Speybroeck, Alexander ${ }^{1,2}$, Jacobs, Robert A. ${ }^{1,2}$, Rivera, Natalie E. ${ }^{3}$, Lee, Jeannie ${ }^{2}$, Saghafi, Saba ${ }^{2}$, To, $\mathrm{Hao}^{2}$, Tran, Tu N. ${ }^{2}$, Nguyen, Benjamin ${ }^{2}$, Alferez, Jose $^{2}$ \\ ${ }^{1}$ USC Keck School of Medicine \\ ${ }^{2}$ Children's Hospital Los Angeles \\ ${ }^{3}$ St. George's University School of Medicine}

Background The HCT service needs of AEA with special health care needs has generated more attention during the past two decades. Widespread international efforts are underway to implement and test the effectiveness of HCT service models. Previous reviews of HCT models focus on the transfer of care rather than on comprehensive HCT services. Many gaps exist in the body of HCT science essential to the development of evidence-based HCT practice.

Methods A retrospective analysis of data extracted from the charts of 146 AEA, ages 9 to 20 who were provided services in the Movin' On Up HCT program in the CHLA Spina Bifida Program from January 1, 2011 to October 15, 2012 are presented. Data were extracted using the 41-item Spina Bifida Extraction Tool composed of demographic and clinical profile; number and type of services provided; number of disciplines involved. Coding of HCT Services was based upon the HCT Research Consortium HCT and Omaha System frameworks.

Results Analyses of services revealed a total of 315 team conferences involving RN (305), MD (268), RD (79), OT (212), PT (207) SW (292) were held, based on these domains: individual (315), family/social support (253); healthcare system (296) and environment (285). Intervention types were teaching, guidance \& counseling (TG\&C) (351); treatments/procedures (T/P) (16); case management (CM) (314) and surveillance (315). A total of 134 direct HCT interventions were conducted addressing following domains: individual (131); family/social support (121); healthcare (97) and environmental (114). Interventions types were TG\&C (131); T/P (4); CM (119); and surveillance (126). 
Conclusions Findings indicate AEA present with a myriad of needs that require a comprehensive HCT service model of care to facilitate not only their transfer of care but movement towards acquisition of developmental competencies of adulthood.

Extending the Adolescent/Young Adult SelfManagement and Independence Scale from Interview to a Self-Report Format

Sawin, Kathleen J. ${ }^{1,2}$, Brei, Timothy ${ }^{3,5}$, Holmbeck, Grayson $^{4}$

${ }^{1}$ Children's Hospital of Wiscosin

${ }^{2}$ University of Wisconsin-Milwaukee

${ }^{3}$ Seattle Children's Hosptial

${ }^{4}$ Loyola University Chicago

${ }^{5}$ University of Washington

Background It is critical for individuals with Spina Bifida, their families, and health care providers to evaluate adolescents' and young adults' (AYA) selfmanagement behaviors in order to transition to a full adult life. Several instruments address the AYA's ability to perform self-management behaviors, but few tap how often the behavior is performed independently. While the 17-Item interview version of the Adolescent/ Young Adult Self-Management and Independence Scale (AMIS II) has been used for several years in research and has solid reliability and validity data, many interdisciplinary professionals have indicated that a self-report (SR) version could have added value in busy clinical practice or in ongoing research. The purpose of this project was to develop the AMIS II in a SR format.

Methods The development of this self-report version of the AMIS II was carried out by a team of four interdisciplinary colleagues who had used the original AMIS II measure. Using an interactive iterative process, the team created, reviewed, and revised items that captured the content of the interview version. Originally the AMIS II was designed to be used in collaboration with the Functional Independence Measure ${ }^{\circledR}$. Thus, items specific to bowel and bladder programs were not included in the AMIS II. However questions to address SB-specific issues were included in the SR/SB version.

Results This iterative process resulted in two SR measures, a 28-item generic AMIS II-SR/G and a 36-item AMIS II-SR/SB. The latter is being used in several centers and data will be pooled for psychometric analysis. Until sufficient sample analysis of the subscales has been collected, item and total scale scores are recommended for the AMIS II-SR/SB.

Conclusions Two SR versions of the AMIS II are now available for field testing. The AMIS II-SR/SB is being evaluated as a preliminary outcome in several programs.

\section{Assessing Readiness for Transition in Adolescents with Spina Bifida \\ Rocque, Brandon G., Graham, Anna, Hopson, Betsy D., Davies, Susan, Blount, Jeffrey University of Alabama at Birmingham}

Background The transition to adult health care for adolescents with chronic disease is crucially important. We have systematically studied readiness for transition in adolescents with Spina Bifida (SB).

Methods We administered the Transition Readiness Assessment (TRA) to 58 adolescents with SB during their annual visit to a multidisciplinary clinic and extracted data from the research electronic medical record associated with the NSBPR. We then constructed linear regression models to examine the relationship between clinical and demographic variables and the proportion of TRA items answered "Yes, I know this." In addition, we created groups of TRA items: medical logistics, knowledge of medical condition, and social/legal concerns, and examined these groups for relationships with clinical variables.

Results The TRA was completed by 54 adolescents, 41 of whom were able to complete it on their own. The majority were white non-Hispanic (69\%), had a diagnosis of myelomeningocele (76\%), and had a CSF shunt (69\%). The mean number of TRA questions answered was 21 of 23. The average proportion of "Yes" answers was 0.58. Adolescents with myelomeningocele as the primary diagnosis and those with CSF shunts had a lower proportion of 'yes' answers ( $0.54 \mathrm{v}$. $0.72,0.55 \mathrm{v}$. 0.66 respectively). Neither of these differences were statistically significant. Questions addressing medical knowledge had the highest mean proportion of "yes" answers (0.81) compared to medical logistics and social/legal questions ( 0.49 and 0.52 respectively).Linear regression modeling revealed a significant association between age and proportion of "yes" answers on questions about medical logistics and social/legal issues. However, this association was not significant when considering only myelomeningocele patients.

Conclusions Administering the TRA to adolescents with SB has revealed areas in which efforts should 
be focused to improve readiness for transition to adult care.

\section{Urological Care for Adults with Spina Bifida: A North American Survey \\ Misseri, Rosalia, Szymanski, Konrad, Swenson, Carly Riley Hospital for Children at Indiana University Health}

Background People with SB require lifetime urologic care. Successful care for adults with SB is dependent on the availability healthcare professionals to provide expert care as well as a system that supports such care. We sought to determine the model in which urologic care is provided to adults in North America.

Methods We administered an online survey to all participants in the Congenitalism Forum at the 2016 AUA meeting. The group includes physicians, allied healthcare professionals and members of health agencies. We collected data on respondents' urologic subspecialty, transition model offered at their institution, method of referral, and level of participation in multidisciplinary clinic. Fisher's exact test was used for statistical analysis.
Results The survey was sent to 208 individuals. Forty urologists (38 US, 2 Canada) responded.

Subspecialty breakdown included female/pelvic floor reconstructive urologists (FPFR): 12.5\%, genitourinary reconstructive urologists(GUR): $20 \%$, pediatric urologists (PU): $67.5 \%$. Continued care and transfer of care were the most common models of transition accounting for $2 / 3$ of responses. Co-management with eventual transfer of care occurs $20 \%$ of the time. PRN care is least common, occurring in $12.5 \%$. Transition models differ based upon the subspecialty of the respondents. Transfer of care is most common for $\operatorname{FPFR}(80 \%)$ and GUR(75\%). Half of PU describe their adult SB practice as continued care model ( $p=$ $0.001)$. More than half (22/40) of PU who responded participate in a multidisciplinary clinic. A minority (7/40) participate in an adult multidisciplinary clinic. Of those participating in an adult clinic $4 / 7$ are PU. The frequency of adult multidisciplinary clinics ranges from weekly (2) to quarterly (1) with most being held monthly (3). Most clinics see 1 to 5 patients per session.

Conclusions There is no uniform mechanism for transition of care and continued urologic care for people with SB in North America. 